\title{
Cumulative Trauma, Personal and Social Resources, and Post-Traumatic Stress Symptoms Among Income-assisted Single Mothers
}

\author{
Joan A. Samuels-Dennis \& Marilyn Ford-Gilboe \&
}

Piotr Wilk \& William R. Avison \& Susan Ray

\begin{abstract}
Data from 247 single mothers were used to partially validate a theoretical model that highlights the process through which post-traumatic stress disorder (PTSD) develops among women. Structural equation modeling was used to assess the direct and indirect relationship between cumulative trauma (CT) and mothers' PTSD symptom severity. Additionally, we examined the meditational role played by mothers' strains and resources and the moderating role played by mothers' residence in an intersectionally advantaged versus disadvantaged neighborhood. A good fit was found between the hypothesized model and data. Mothers' strains and personal resources played a significant mediating role in the relationship between CT and PTSD symptom severity. Neighborhood of residence did not moderate the CT-PTSD process. Implication for practice and treatment are discussed.
\end{abstract}

Keywords Intersectionality. Post-traumatic Stress symptoms . Psychological and assaultive trauma. Single mothers

This research was supported by a doctoral fellowship from the Ontario Women's Health Council (OWHC)/Canadian Institute of Health Research (CIHR) awarded to Joan Samuels-Dennis.

J. A. Samuels-Dennis (*)

Faculty of Health Studies, School of Nursing, York University,

4700 Keele Street.

Toronto, ON M3J 1P3, USA

e-mail: jsdennis@yorku.ca

M. Ford-Gilboe : S. Ray

Nursing, University of Western Ontario,

London, ON, Canada

P. Wilk W. R. Avison

Sociology, University of Western Ontario,

London, ON, Canada
Among general population studies conducted in Canada and the United States it has been demonstrated that women are twice as likely to experience lifetime post-traumatic stress disorder (PTSD) when compared to men (Breslau et al. 1999; Stein et al. 1997). Furthermore, as we begin to look at sub-groups of women, we also observe that some groups experience more advantage or disadvantage in terms of their lifetime risk for PTSD. Among women who are mothers, married mothers appear to be the most advantaged with $8 \%$ experiencing lifetime PTSD (Afifi et al. 2006). While homeless and income-assisted single mother appears to be the most disadvantaged with about $30 \%$ experiencing PTSD over their lifetime (Bassuk et al. 1998; Tolman and Rosen 2001).

In an earlier publication we introduced the intersectionality model of trauma and PTSD (IMT-PTSD; SamuelsDennis et al. 2009b), a framework that attempts to accounts for these observed disparities in mental well-being. The model (Fig. 1) integrates current empirical literature with principles of intersectionality (Crenshaw 1994) and the stress process formulation (Pearlin 1999) and outlines a social process through which PTSD develops and persists among women. Using the IMT-PTSD model, we suggest that mental health is determined by macro-level factors (neighborhood structural and political characteristics) that intersect to shape power inequalities, discriminating social relations, and the distribution of health and social resources. In turn, these intersecting factors facilitate a meso-level trauma-PTSD process, whereby, gender-based traumanon-naturally occurring traumatic experiences that are motivated and supported by entrenched beliefs about the socially ascribed roles, responsibilities, and locations of individuals belonging to the male and female sex-directly and indirectly impact women's mental health and wellbeing through its influence on women's personal resources 
Fig. 1 Intersectionality Model of Trauma and PTSD
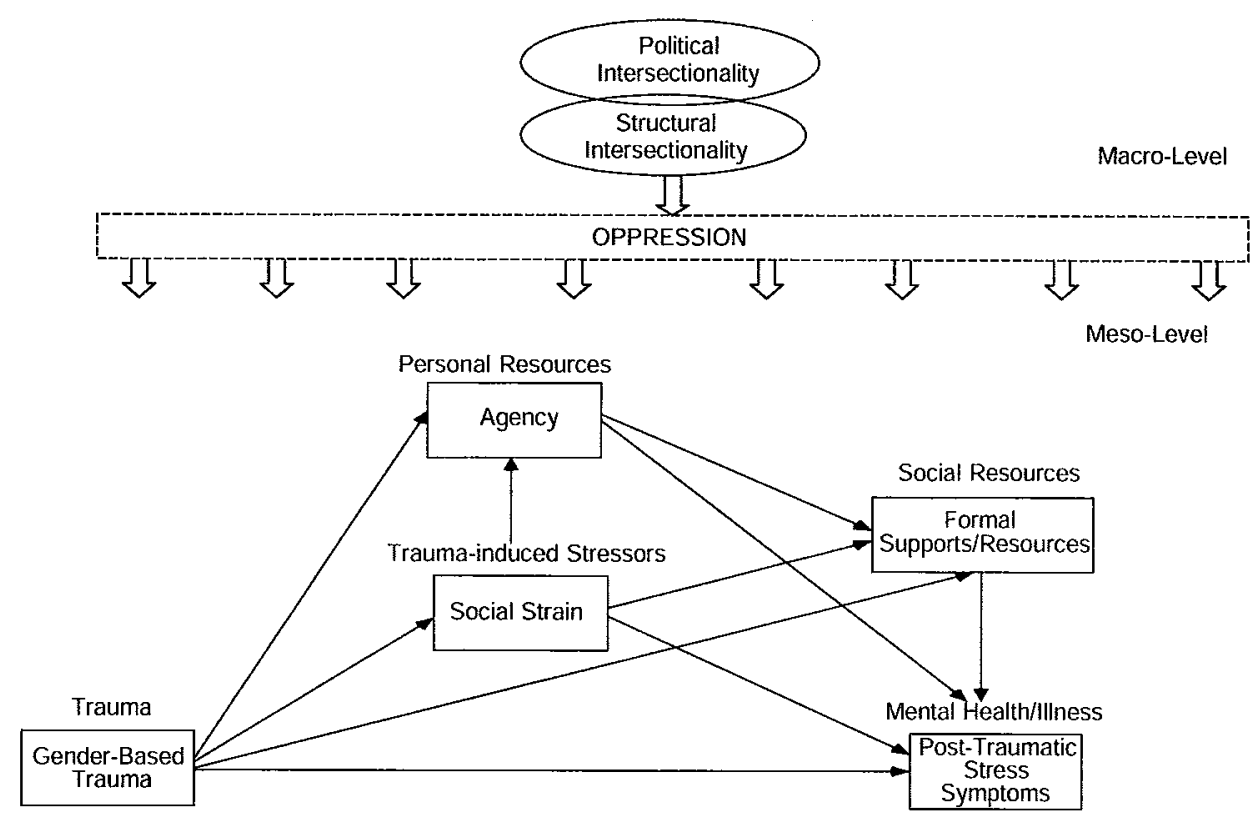

(agency), social resources (formal support/resources), and trauma-induced interpersonal stressors (social strain).

The current study was undertaken to partially validate the IMT-PTSD model among a sample of income assisted single mothers. As illustrated by Fig. 2, we consider this analysis a partial test because we examine the direct relationship between cumulative adversity and trauma (CAT) and mothers' PTSD symptom severity and the mediating roles played by trauma-induced stressors (social strain), personal resources (personal and interpersonal agency), and formal supports/resources (primary and specialized mental health service use). This is also considered a partial test because the IMT-PTSD model calls for an examination of the direct and indirect relationship among the mediators in the model (strains and resources), but the sample size of 247 did not allow for such a complex analysis. In this analysis, we instead control for the inter-relationship among mothers' strains and resources.

Theoretical Model and Review of the Literature

The IMT-PTSD model (Samuels-Dennis et al. 2009a, b) situates PTSD within a social determinant of health perspective and suggests that disparities in mental wellbeing reflect both the intersection between people's diverse
Fig. 2 Theoretical Model derived from the Intersectionality Model of Trauma and PTSD
Neighbourhood (Intersectionality) Status

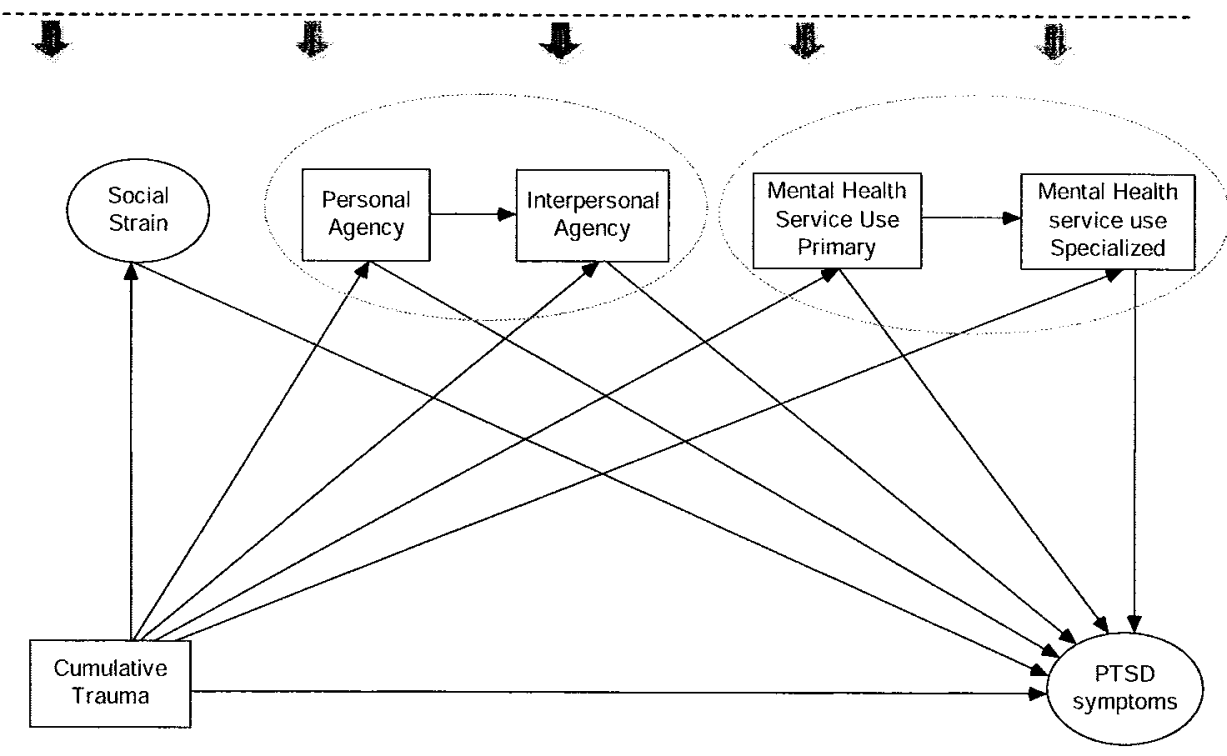


experiences and the social context of their lives. The model purposefully focuses on the structural and political context of women's lives with the explicit understanding that people's multiple and intersecting social statuses defines the conditions of life to which people are exposed. An underlying premise of the IMT-PTSD model is that neighborhoods provide a geographic and social space whereby, the structural and political characteristics of neighborhoods supersede individual social and economic status in determining the types and sources of trauma/ stressors/strains to which residents are exposed, as well as the resources available for coping with, and managing the impact of difficult life experiences.

The central determinant of mental well-being is the social dis/advantage people experience as a direct consequence of intersectionality-multiple and interconnected structural and political neighborhood characteristics. Neighborhood in its broadest sense refers to geographic boundaries including countries, provinces/territories/states, cities, and regions. Within neighborhoods, structural intersectionality attends to co-relating systems of oppression that are supported by socially ascribed identities (i.e. gender, economic status, race/ ethnicity, family structure, dis/ability, and sexual orientation). In contrast, political intersectionality highlights how the political interests of various groups within neighborhoods are obscured and sometimes jeopardized by political dialogue, legal directives and government policies that ignore or suppress issues of group-related disadvantage.

The structural and political characteristics of neighborhoods encompass a social location best described as intersectionally advantaged or disadvantaged with significant implications for the trauma-PTSD process. Although we are unaware of studies that address variations in the trauma-PTSD process across intersectionally advantaged/ disadvantaged neighborhoods, a growing body of research suggests that people living in intersectionally disadvantaged neighborhoods experiences greater lifetime exposure to violence (Cunradi et al. 2000), reduced access to health and social resources (Allard et al. 2003), and poorer mental well-being (Galea et al. 2007).

\section{The Trauma-PTSD Process}

The social process outlined in Fig. 2 depicts the proposed relationship between five variables: 1 ) cumulative adversity and trauma (CAT), 2) social strain, 3) agency (personal and interpersonal), 4) mental health service use (primary and specialized), and 5) PTSD symptom severity.

\section{Cumulative Adversity and Trauma (CAT)}

In this study, CAT refers to the phenomena whereby exposure to one set of trauma or adversity gives rise to other negative and detrimental experiences, the effects of which surface and recede or function in combination to impact mental health (Pearlin 1999). In using this conceptualization we acknowledge two things: First that all life experiences, whether adverse or traumatic, matter to mental well-being. Second that the potential impact of any given experience shifts with the context of women's lives. That is, a cumulative effect in the trauma-PTSD process will be determined by a number of factors including: 1) the number of unique events people experience over their lifetime, 2) the nature of the event and the multiplicity of exposure, and 3) the enduring or chronic nature of the traumatic event(s) (Breslau et al. 1999; Kaysen et al. 2003).

Among the spectrum of adverse and traumatic events that may precipitate PTSD in women, the current study places an emphasis on accounting for the effects of assaultive and psychological traumas. Assaultive traumas include discrete and/or enduring offensive physical and sexual encounters that precipitate a state of intense fear, apprehension, loss of control, and powerlessness in victim (Samuels-Dennis et al. 2009a, b). While psychological traumas include enduring, uncontrollable, and emotionally overwhelming, encounters in which a perpetrator uses fear, intimidation, and manipulation to render an individual powerless (Samuels-Dennis et al. 2009a, b). The importance of assaultive and psychological trauma is supported by three recent studies (Breslau et al. 1999; Coker et al. 2000; Pico-Alfonso 2005) which suggest that women's 3:1 greater risk for lifetime PTSD when compared to men is due to their greater lifetime exposure to psychological and assaultive trauma.

Given that single mothers on social assistance experience higher rates of childhood physical and sexual abuse, physical assault by strangers, and intimate partner violence (IPV) (Bassuk et al. 2006; Schumm et al. 2006; Tolman and Rosen 2001), a gender-sensitive analysis of the social process through which PTSD develops among single mothers must account for the cumulated effects of assaultive and psychological events that, although not discrete or short-lived events, are nevertheless outside the range of usual human experience.

\section{Social Strain}

Particularly for women subjected to prolonged and repeated psychological and/or assaultive trauma(s), the IMT-PTSD model identifies social strain as one of the more significant consequences of trauma exposure as it represents a social context characterized by the chronic absence of social support and/or consistent problematic interactions with members of one's social network. Social support has generally been conceptualized as a personal or social resource that mediates or moderates the relationship 
between stress and mental health problems. However, recent studies (Andrews et al. 2003; Coker et al. 2002; Hyman et al. 2003; Schumm et al. 2006; Ullman et al. 2007) identify the unavailability of social support and qualitatively poor interactions with one's social network as important factors in the trauma-PTSD process.

Coker et al. (2002), examined the relationship between received support versus absent or inconsistent support from friends, family, and a current non-abusive spouse and PTSD symptoms in a sample of women $(N=1,152)$ previously exposed to IPV. Their findings revealed that receiving consistent high versus low levels of emotional support from any of the three sources reduced women's PTSD symptom levels, with emotional support from friends having the greatest effect. Addressing the question of how various dimensions of social support (availability of others, confiding in others, emotional support, practical support, negative responses, and satisfaction with support) explain PTSD symptom severity, Andrews et al. (2003) found that negative responses to women's assault experiences was the only significant predictor of PTSD symptoms 6-months post physical or sexual assault.

Beyond consistent support and negative interactions, recent research also suggests that the absence of specific types of social support also affect PTSD symptom severity. In a sample of 172 sexual abuse survivors, Hyman et al. (2003) found that self-esteem support-the perception that others value the individual, and appraisal support-the perception that one is able to obtain advice when coping with a problem, were inversely associated with survivors' symptom levels. Although it has not been systematically examined, current literature suggests that trauma exposure gives rise to social context whereby trauma-exposed individuals experience chronically low levels of support and persistent heightened levels of interpersonal conflict, that, in-turn, heighten PTSD symptom severity. The current study addresses this gap in knowledge by examining the mediating role of social strain the CAT-PTSD process.

\section{Agency}

Agency is a personal resource and represents people's capacity for appraising and assigning meaning to their life circumstances, making meaningful choices, and their ability to engage in activities that create personal, social and political change (Bandura 2001; Benight and Bandura 2004; Samuels-Dennis et al. 2009a, b). In this study, we focus on two inter-related agentic outcomes-personal and interpersonal agency-and examine their mediating role in the trauma-PTSD process.

Various operationalization of agency can be found in current literature. However, (Bandura 2001; Benight and Bandura 2004) suggest that studies of self-efficacy may provide the most comparable insights regarding the protective influence of agency in the post-trauma period. Among the many dimensions of human agency, they propose that none is more central than people's beliefs in their efficacy to manage their own functioning and to exercise control over events that affect their lives. The mediating role of self-efficacy in the trauma PTSD process has been examined across a range of traumatic experiences including people exposed to combat (Ginzburg et al. 2003), physical injury caused by non-domestic violence (Johansen et al. 2007), and child abuse (Vranceanu et al. 2007). Results of these studies have consistently shown that exposure to trauma diminishes levels of perceived selfefficacy which, in turn, elevates PTSD symptoms.

Mental Health Service Use

The IMT-PTSD model highlights the important role of formal supports and resources, including legal services, housing, income assistance, and health services, as mediators in the relationships between trauma and women's mental health. In this study, we focus specifically on the role of mental health service use in the trauma-PTSD process. Previous research has consistently shown that exposure particularly to assaultive traumas increases women's use of mental health services. For example, Rivara et al. (2007) found that, compared to women with no history of intimate partner violence (IPV), women who experienced IPV were more likely to use mental health services during and after their IPV experiences. Similar findings have also been reported among women exposed to sexual assault and victims of violent crimes (Elhai and Simons 2007; Lewis et al. 2005).

Although it has been established that both trauma exposure and PTSD symptom severity influence health service use, a significant gap in the literature is the absence of research that examines whether increased use, particularly of specialized mental health services, influences the resolution of mental health problems. Fundamentally, use of services does not necessarily ensure that quality care is received. However, given that guidelines for the treatment of anxiety disorders have been developed and are available to mental health service providers (see Stein 2004), it is reasonable to investigate whether use of mental health service impacts PTSD symptom severity.

\section{Hypothesis}

Informed by previous research and the IMT-PTSD framework, a causal model was constructed to assess the effect of CAT, current strain, and resources on mothers PTSD symptom severity (Fig. 2). The model contains three latent 
variables, each having three indicators, and four manifest variables. CAT is hypothesized to affect PTSD symptom severity directly and indirectly, through its impact on women's agency, level of social strain, and use of mental health services. CAT is proposed to: a) increase women's experiences of social strain, leading to more severe PTSD symptoms, b) increased use of mental health services (primary care and specialty services), resulting in less severe PTSD symptoms, and, c) erode women's personal and interpersonal agency, leading to more severe PTSD symptoms. Finally, we hypothesize that this process will be moderated by women's neighbourhood of residence which is used as a proxy for intersectional advantage and disadvantage.

\section{Method}

Sample

Participants $(\mathrm{N}=247)$ for this cross-sectional study were recruited from the active caseload of the provincial social assistance program. The eligibility criteria included: 1) female caregiver for one or more dependent child $(<18$ years old); 2) receipt of income-assistance for 6 or more months; 3) living without a spouse/common law partner; and, 4) fluent in English. The average age of participants was 35.7 years ( $S D=9.5$, Range $18-61$ years).

\section{Recruitment \& Data Collection Procedure}

A total of 2,400 single mothers randomly selected from the active caseload of the social service agency were contacted by mail between January and February, 2008, to request their participation in the study. In total, 293 single mothers contacted the principal investigator by telephone, mail, and e-mail to obtain information about the study, 10 of whom decided not to take part in the study. Of the 283 remaining, 271 single mothers met the eligibility criteria. Ninety-five percent of those found eligible to participate completed a telephone survey with a trained interviewer $(n=173)$ or online $(n=82)$ survey designed to assess lifetime exposure to adversities and traumas, current symptoms of PTSD, use of health services, personal and social resources, and selected demographic characteristics. Sixteen eligible participants did not complete the survey and incomplete data resulted in eight surveys being excluded from the final analysis. A detailed description of the sample, recruitment process, and reasons for the low response rate are reported elsewhere (Samuels-Dennis et al. 2009a).

More than half (56.1\%) of the sample were single-nevermarried mothers while $42.4 \%$ were separated or divorced. Caucasians made up the largest proportion of the sample (37.7\%) followed by Blacks (28.5\%), Hispanics (7.5\%),
South Asians (6.7\%), and biracial mothers (7.5\%). The group was highly educated with just fewer than $49 \%$ having participated in some post-secondary education. Although actively receiving social assistance $22 \%$ of the sample was employed either part-time or full-time. Forty-six percent of the sample immigrated to Canada.

\section{Measures}

Cumulative Adversity and Trauma (CAT)

The latent variable CAT was constructed from three indicators: 1) lifetime adversity exposure, 2) lifetime psychological trauma exposure, and 3) lifetime assaultive trauma exposure. After reviewing the 23 items presented in Table 1, participants indicated if they had ever experienced the event. If lifetime exposure was confirmed, participants were next asked to indicate how many times they experienced each event during childhood ( $<18$ years) and adulthood (• 18 years). Responses were coded 0 (no exposure) or 1 (at least 1 occurrence) for each developmental stage. Exposure scores were calculated using the following procedure: First, the 11 adversity, 4 psychological, and 8 assaultive items for childhood and adulthood were summed. Second, the childhood and adulthood exposure scores were transformed to z-scores so that events in each category and developmental stage had equal weighting. Third, childhood and adulthood z-scores for each category of events were summed to create a lifetime exposure score.

\section{Social Strain}

The latent variable social strain was constructed from three indicators: 1) total scores from the self-esteem and appraisal support sub-scales of the Interpersonal Support Evaluation List (ISEL: Cohen et al. 1985), and, 2) total scores for the social conflict subscale of the Interpersonal Relationship Inventory (IPR: Tilden et al. 1990). The ISEL is a 40-item summated rating sale developed to measure the perceived availability of tangible, appraisal, self-esteem, and belonging support. We used only the self-esteem support [having someone with whom to discuss issues of personal importance (10 items)] and appraisal support [the presence of someone with whom the individual feels he/she compares favorably (10 items)] subscales as previous studies demonstrated a relationship between these forms of support and PTSD symptom severity. Participants rated the truthfulness of each item on 4-point likert scale ( $0=$ definitely true, $3=$ definitely false). Item scores were reverse coded and the sub-scale items summed. Scores ranged from 0-30 with higher scores indicating lower perceived self-esteem and appraisal support. Validity and reliability has been demon- 


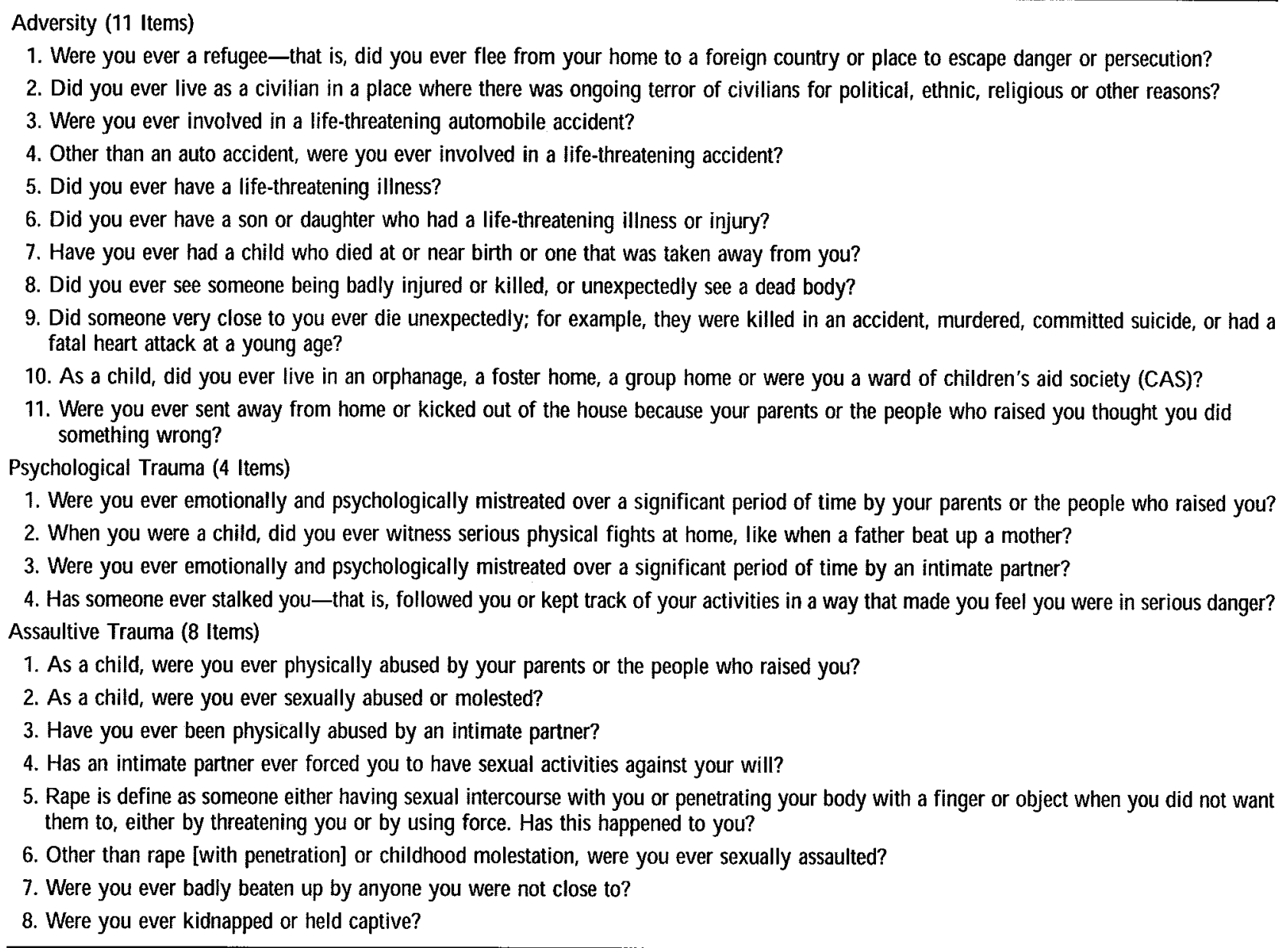

Items were derived from the Composite International Diagnostic Interview-PTSD module (World Health Organization 2004) and the Betrayal Trauma Survey (Goldberg and Freyd 2006)

strated among undergraduate students and women exposed to assaultive trauma (Cohen et al. 1985; Hyman et al. 2003) with Cronbach's alpha coefficients ranging from .60 to .68 and .77 to .92 for the appraisal and self-esteem subscales respectively. In this study, Cronbach's alpha for the appraisal and self-esteem subscale were .68 and .73, respectively.

The social conflict subscale of the IPR (Tilden et al. 1990) is a 13-item self-report inventory designed to measure perceived degrees of conflict in interpersonal relationships. Participants rated how often each conflict item occurred on a 5 -point likert scale ( $1=$ never and $5=$ very often). Scores (range 13-65) were derived by summing the 13 items, with higher scores indicating greater interpersonal conflict with members of one's social network. Subscale reliability has demonstrated in samples of chronically ill, homeless, and IPV-exposed women (Anderson and Rayens 2004; Goodwin 1997) with Cronbach's alpha ranging from .81 to .92 . For this study, Cronbach's alpha was .89 .

\section{Agency}

Personal and Interpersonal agency were measured using the 8-item personal agency scale (PAS) and the 5-item interpersonal agency scale (IAS) (Smith et al. 2000). Both measures assess the frequency with which individuals use specific strategies to acquire control over their life circumstance and achieve personal goals. Personal agency refers to specific acts that are performed intentionally to achieve a desired outcome, while interpersonal agency is a social process in which individuals purposefully communicate and collaborate with those holding formal and informal power to acquire their assistance in achieving a desired outcome (Smith et al. 2000). Participants identified how often on a 4point likert scale $(1=$ never to $4=$ often) they engaged in 13 specific behaviours such as 'I achieve my goals by knowing when to ask others for help'. PAS (range 8-32) and IAS (range 5-20) scores were derived by summing the appropriate items, with higher scores indicating greater 
perceived agency. Cronbach's alpha for the PAS and the IAS in one previous study were .76 and .78 , respectively, indicating adequate internal consistency (Smith et al. 2000). For this study Cronbach's alpha for the PAS and IAS were .85 and .83 , respectively.

\section{Mental Health Service Use}

Primary mental health service use (MHSU-P) was measured by the total number of self-reported telephone or in-person visits made to six care providers (family doctor, public health nurse, psychiatric/mental health nurse, nurse practitioner, telephone crisis worker, and child protection worker) because of a mental health problem in the past 3 months. These providers act as a first point of consultation regarding a mental health concern and are authorized to refer patients on for specialized mental health care. Specialized mental health service use (MHSU-S) was measured by the total number of self-reported telephone or in-person visits made to 6 mental health specialist (i.e. psychiatrist, psychologist, social worker, counsellor other than social worker, and clinical nurse specialist) to treat and/or manage a mental problems in the past 3 months.

\section{PTSD Symptom Severity}

The 17-item Davidson Trauma Scale (DTS; Davidson 1996) assessed the intensity of three clusters of PTSD symptoms experienced over a 1-week period. Participants first reviewed the definition of a traumatic event and identified their exposure/non-exposure to a lifetime trauma. Participants who reported no trauma exposure $(\mathrm{N}=67)$ were assigned a DTS score of zero. Those who reported a lifetime trauma $(\mathrm{N}=180)$ identified on a 5-point likert scale the frequency $(0=$ not at all to $4=$ every day) and severity $(0=$ not at all distressing to $4=$ extremely distressing) of their post-traumatic symptoms. Score were calculated by summing the appropriate frequency and severity items for the intrusion (range 0-40), avoidance/numbing (range 0-56), and hyper-arousal (range 0-50) symptom clusters. Excellent reliability for the full scales has been demonstrated among victims of violence (Bradley et al. 2005; Davidson 1996) with Cronbach's alpha coefficients ranging from 0.79 to 0.99 . In this study, Cronbach alpha's coefficients were .94 , .94 , and .93 for the intrusion, avoidance/numbing, and hyperarousal subscales, respectively.

Neighborhood (Intersectionality) Status

Although it is difficult to fully address all features of intersectionality, recent trends in the collection of census tract data and the political direction of some cities in purposefully acknowledging geographic areas within their borders that fare better or worst in terms of resource allocation, provide, a means of quantitatively examining how neighborhood structural and political characteristics intersect to impact mental health. The City of Toronto recently identified 13 priority neighborhoods. The term priority implies that the neighborhood is inadequately resourced and the need for formal political interventions that will increase residents' access to community resources. While disparities in the allocation of resources explicitly characterize these neighborhoods, what is less explicit are the ways in which these neighborhoods differ structurally. Compared to the larger metropolis, priority neighborhoods vary, on average, by a number of intersecting structural characteristics including: 1) a $1.5 \%$ greater proportion of persons age $15-24(14.0 \%$ vs. $12.5 \%)$; 2) $16.4 \%$ more immigrant families $(65.8 \%$ vs. $49.4 \%)$; 3 ) $15.7 \%$ more visible minorities (58.6\% vs. $42.8 \%$ ); 4) $6.8 \%$ more lowincome persons $(29.4 \%$ vs. $22.6 \%)$; 5 ) $\$ 18,439$ lower annual household income $(\$ 40,686$ vs. $\$ 69,125)$; and 6 ) $4.3 \%$ more lone parent households (24.0\% vs. $19.7 \%$ ) (City of Toronto 2006).

In this analysis we suggest that the structural and political characteristics of priority and non-priority neighborhoods encompass a social location that serves an adequate proxy for intersectional advantage and disadvantage. Thus Mapped boundaries for the 13 prioritized neighborhoods were used in conjunction with participant's addresses to identify whether participants lived in a priority (code $=1$ ) or non-priority (code $=0$ ) neighborhood. Thirtyfive percent of the sample $(n=87)$ lived in a priority neighborhood. The groups did not differ in terms of age, \# of children, marital status, race/ethnicity, education, and employment status. However, a significantly greater proportion of priority neighborhood residents (58.6\%) immigrated to Canada compared to their non-priority (39.0\%) counterpart $\left(x^{2}=8.86, d f=1, p=.002\right)$.

\section{Data Analysis Strategy}

Data were analyzed using SEM in AMOS 7.0 (Arbuckle 2006) with the maximum likelihood (ML) method of estimation. SEM represents a mix of both path and confirmatory factor analytic procedures, involving the comparison of hypothesized model covariances with observed covariances (Kline 2005). Advantages of this procedure include the flexibility of model specification and the ability to assess fit of the hypothesized model to the observed data (Kline). The analysis proceeded in two stages. We first tested the validity of model for the entire sample and then completed a multi-group analysis which tested for variations in the model structure across neighborhood groupings. 
The goodness of fit between the hypothesized model and the data obtained from this sample was assessed by five fit indexes using established critical values including: 1) a non-significant $(p>.05)$ chi-square statistic $\left(x^{2}\right)$ (Jöreskog and Sörbom 1995), a normed chi-square statistic $\left(x^{2} / d f\right)$ $<2.00$ (Byrne 2001), comparative fit index (CFI) $\cdot .95$ (Bentler 1990), Parsimony-Adjusted CFI (PCFI) 0.50 (James et al. 1982), and a root mean square error of approximation (RMSEA) • .05 (Browne and Cudeck 1993).

Multivariate normality, multivariate outliers, linearity, homoscedasticity, and multicolinearity were assessed prior to analysis. This revealed that the distribution for MHSU-P (skewness = 7.61, kurtosis = 71.42) and MHSU-S (skewness= 5.42, kurtosis $=35.93$ ) were problematic. Because SEM assumes all variables are normally distributed these variables were transformed using the formula $-1 /(x+c)$. This transformation significantly improved the distribution for both variables (MHSU-P: skewness $=.039$ and kurtosis $=1.34$, MHSU-S: skewness $=\cdot 1.77$, kurtosis $=.032$ ). Additionally, 3 of 247 participants failed to provide responses to all items. Because AMOS 7.0 fails to provide modifications indices, assessments of normality, and significance levels for direct and indirect relationships when data are missing, these cases were deleted prior to the final analysis.

Results

Model Fit

Initial analysis revealed a good fit between the hypothesized model and data $\left(x^{2}=47.6, d f=29, p=.02, x^{2} / d f=1.64\right.$, $\mathrm{CFI}=.98, \mathrm{PCFI}=.52$, RMSEA $=.05$ ). Thus, the proposed model accounted adequately for the observed variance, covariances, and error co-variances among the indicators and provided support for the hypothesized model.

\section{Measurement Model}

Descriptive statistics for the indicators used in the measurement model are presented in Table 2. Inspection of the parameter estimates of the model and their associated critical ratio values provided support for the hypothesized structure of the measurement model. The factor loadings were all statistically significant and were of substantial magnitude (.48-.94). There were no unreasonable parameter estimates, such as negative variances or factor loadings greater than one (Fig. 3).

Direct and Indirect Effects

Figure 4 presents the standardized regression coefficients for the structural model. These path coefficients represent the "pure" relationships between each set of variables in the model, after controlling for the effects of all other variables. Both the direct $(B=.37, p<.01)$ and indirect $(B=.20, p<.001)$ effect of CAT on mothers' PTSD symptom levels were significant, with the direct effect being almost twice as large as the indirect effect. While CAT appears to be the central explanatory variable, the findings also suggest that social strain and agency mediate this relationship. As expected, CAT increased women's experiences of social strain $(B=.40$, $p<.001)$, leading to more severe PTSD symptoms $(B=.53$, $p<.001)$. Through its direct effect on personal agency $(B=$ -.17, $p=.02)$ and its indirect effect on interpersonal agency $(B=\cdot .07, p=.02)$, CAT eroded womens' agency, leading to more severe PTSD symptoms $(B=.16, p=.01)$. The findings related to mothers' use of mental health services (primary and specialty services), were not as expected. CAT had a significant direct effect on mothers' use of primary mental health services $(B=.27, p=.00)$ and indirectly effected mothers' use of specialized services $(B=.07, p=.00)$ through MHSU-P. However neither, primary $(B=.05, p=.19)$ nor specialized $(B=.07, p=.19)$ mental health service use affected PTSD symptom severity. Review of the squared multiple correlations revealed that $51.9 \%$ of the variance in mothers' PTSD symptoms levels was explained by the combination of CAT, social strain, personal and interpersonal agency, and use of primary and specialized mental health services.

\section{Neighborhood Multi-group Analysis}

To test for structural and measurement model invariance across neighborhood groupings, simultaneous goodness of fit tests were completed with three models. The first model allowed all parameter estimates to be different (Model A). The second model constrained all measurement paths across the two groups (Model B). The third model constrained all structural paths across the two groups (Model $\mathrm{C}$ ). The result of the chi square difference test between Model $A$ and $B$ supported the null hypothesis of measurement model equivalence across neighborhood groupings ( $\#_{\text {difference }}^{2} \cdot 1: 01, \mathrm{df}_{\text {difference }}=6, p=68$ ). Similarly, result of the chi square difference test between Model $B$ and $C$ supported the null hypothesis of structural model equivalence across neighborhood groupings $\#_{\text {difference }}^{2} \cdot 17: 01$, $\mathrm{df}_{\text {difference }}=13, p=.20$ ). Thus, as a whole, the relationships outlined in the causal model were not moderated by mothers' neighborhood of residence.

Although the model structure did not vary by neighborhood grouping, independent examination of the model and the paths within, for each group revealed some remarkable findings (Fig. 5). For both groups, CAT directly and indirectly affected severity of PTSD symptoms. However, the mediating variables were different in each group. For 


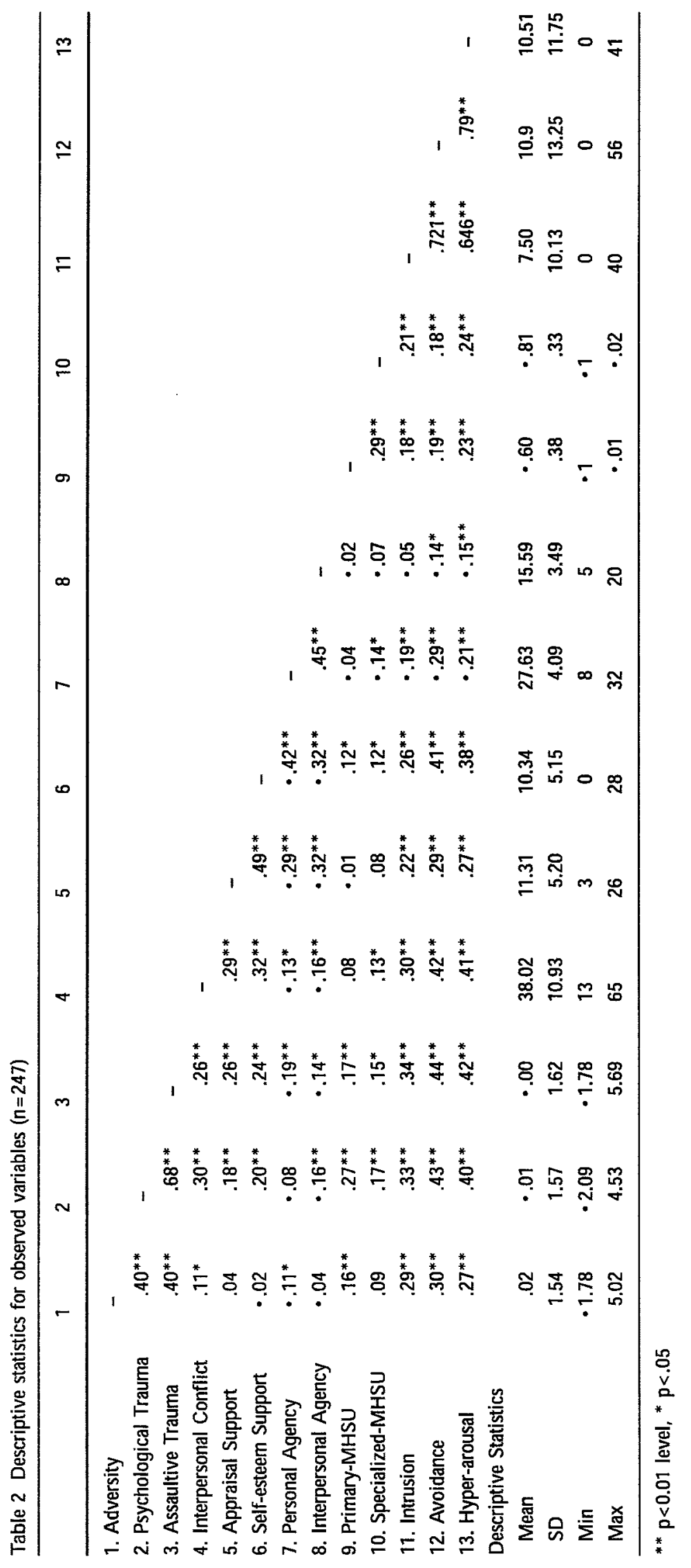


Fig. 3 Hypothesized model with latent variables and indicators. $D=$ variance in endogenous latent variable not accounted for in the model

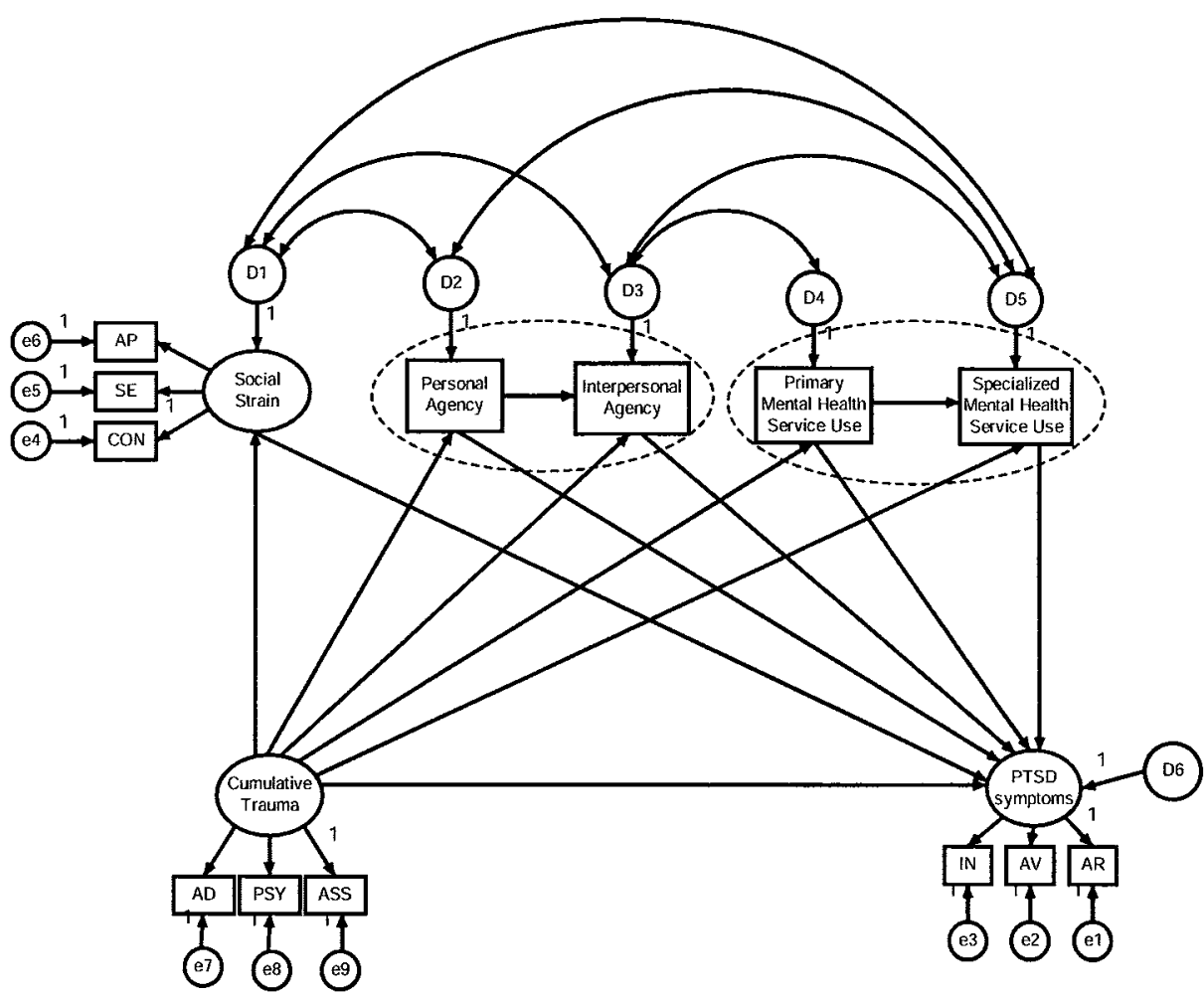

\begin{tabular}{llc}
\hline Latent Variable & Indicators & Factor Loadings \\
\hline CAT & Adversity (AD) & .490 \\
& Psychological Trauma (PSY) & .834 \\
& Assaultive Trauma (ASS) & .813 \\
\hline Social Strain & Self-esteem support (SE) & .738 \\
& Appraisal Support (AP) & .620 \\
& Interpersonal conflict (CON) & .483 \\
\hline PTSD Symptoms & Intrusion (IN) & .764 \\
& Avoidance (AV) & .938 \\
& Hyperarousal (AR) & .848 \\
\hline
\end{tabular}

Note: $D=$ variance in endogenous latent variable not accounted for in the model women living in priority neighborhoods, social strain mediated the relationship between CAT and PTSD symptom severity. CAT increased women's experiences of social strain $(B=.48, p=.01)$, leading to more severe PTSD symptoms $(B=.43, p=.02)$. No other variables mediated the relationship between CAT and women's PTSD symptom severity. For women living in non-priority neighborhoods, both social strain and agency mediated the relationship between CAT and PTSD symptom severity. CAT increased women's experiences of social strain $(B=.48, p=.01)$, leading to more severe PTSD symptoms $(B=.59, p<.01)$. CAT eroded women's personal agency directly $(B=\bullet .20, p<.05)$ and interpersonal agency indirectly $(B=\cdot .07, p=.03)$, leading to more severe PTSD symptoms $(B=.23, p=.02)$. While CAT had a significant direct effect on mother's use of primary
$(B=.24, p=.004)$ and specialized $(B=.15, p=.049)$ mental health service use, mental health service use was not related to mothers PTSD symptom levels.

\section{Discussion}

Traumatic experiences affect the severity of single mother's PTSD symptoms through a variety of pathways. The present study addressed current gaps in knowledge relevant to the relationship between trauma and PTSD symptoms by testing a theoretical model that examines the process through which cumulative adversities and trauma (CAT) affects PTSD symptom severity. Although past studies (Coker et al. 2003; Ford-Gilboe et al. 2009; Schumm et al. 2006; Ullman et al. 
Fig. 4 Standardized path coefficients-Direct effects for the hypothesized model. ${ }^{*} \mathrm{p}<.05,{ }^{* *} \mathrm{p}<.01,{ }^{* * *} \mathrm{p}<.001$

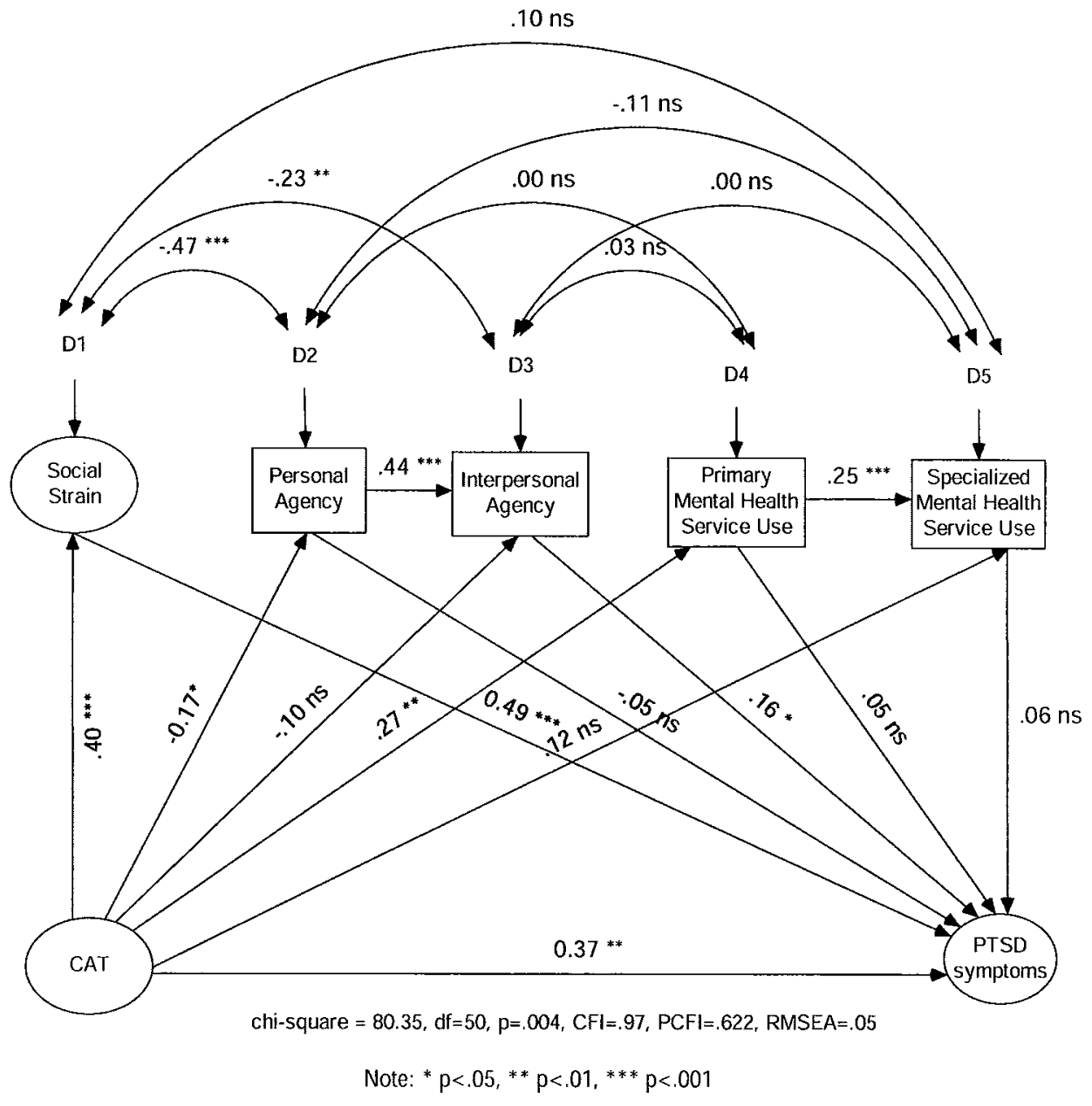

2007) have examine the inter-relationships between some of the constructs presented in this study, the validity of the theoretical model presented here has not been previously tested. The model fit for the full group analysis provided initial support for the causal model. Furthermore, the findings remain consistent with previous research and the broader theoretical perspective that guides this research.

Overall Test of the Model

As hypothesized, CAT exerted a significant direct and total indirect effect on PTSD symptom severity. The direct effect of CAT on PTSD symptom severity was significantly larger than that reported in other studies using SEM to test the impact of physical IPV $(B=.23$; Coker et al. 2003), physical and non-physical IPV $(B=.19$; Ford-Gilboe et al. 2009), sexual assault severity ( $B=.14$; Ullman et al. 2007) on mental health problems including PTSD symptom. Importantly, this analysis moves beyond the traditional focus on a single event or event(s) characterized by assault or threats to physical integrity and includes adversities, psychological, and assaultive traumas. Although the mea- sure could be further developed to assess severity in addition to exposure, this measurement approach allowed for a gender-sensitive operationalization of the construct which attended to the traumatic experiences women are likely to face over their lifetime. The strong effect found here reinforces the growing belief that all experiences matter to the trauma-PTSD process in that a wide range of adverse and traumatic experience occurring in early and later life combine to impact single mothers' mental health (Lloyd and Turner 2003). Furthermore, the cumulated effects of these experiences are far reaching given that they impinge not just on mental well-being, but on the social context of income-assisted single mothers' lives.

As anticipated, cumulative trauma increased single mothers' exposure to social strain, eroded personal and interpersonal agency, and increased single mothers' use of primary and specialized mental health services. Although primary and specialized mental health care did not affect the severity of women's PTSD symptoms, social strain and agency proved to be important mechanisms through which CAT affected women's PTSD symptom levels. The most notable finding was the significant mediating role played by 
Fig. 5 Standardized path coefficients for priority \& nonpriority neighborhoods
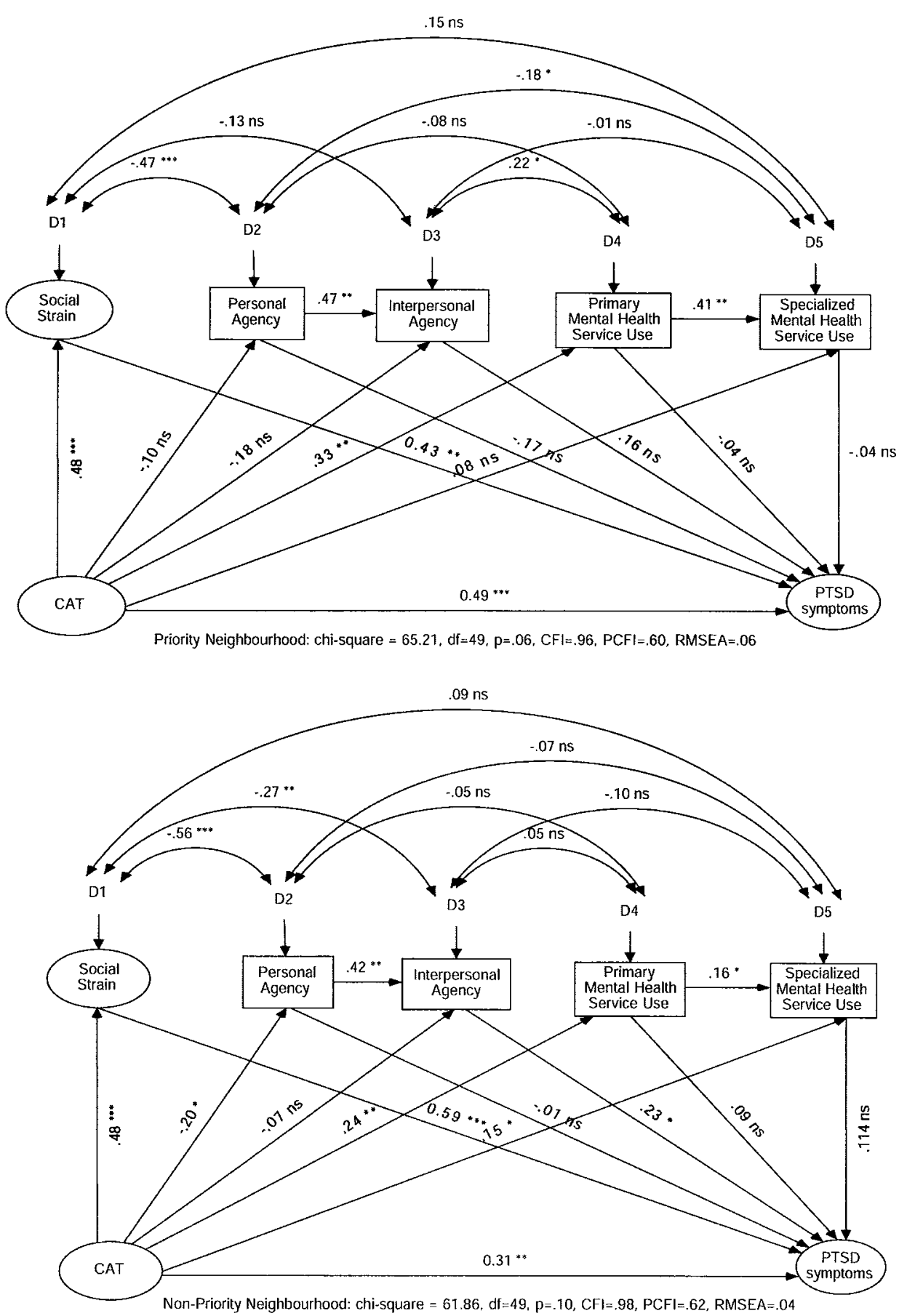

social strain in the CAT-PTSD process. This finding varies from that reported by (Ford-Gilboe et al. 2009). They used SEM to examine the mediating role of social resources (support and conflict) on the relationship between trauma severity (physical and non-physical IPV) and mental health (depression, general functioning, and PTSD). Although they found a significant direct effect of IPV severity on mental health and social resources on PTSD, IPV severity did not affect women's social resources. It is possible that their focus on emotional and tangible support and our focus on self-esteem and appraisal support accounts for this difference. Previous research has shown that tangible and emotional supports do not influence PTSD symptoms severity (Andrews et al. 2003; Hyman et al. 2003). It is also possible that the findings differ because our analysis assessed single mothers' exposure to 23 events both in childhood and adulthood, rather than severity of IPV perpetrated by women's most recent abusive partner. We 
suspect also that the trauma history profiles for the two samples may differ substantially.

Our findings suggest that this vulnerability also extends to social strain. It appears that the breadth of single mothers' experiences and/or the absence of trauma resolution, alters their capacity to form and maintain close, trusting relationships, thus, creating a social context characterized by depleted support and heightened conflict leading to more severe PTSD symptoms. Moving beyond this individualized perspective, it is also possible that a number of intrusions (i.e. interactions with familial perpetrators of abuse and violence, abuserelated health complications, being on social assistance) exacerbate social strain (Ford-Gilboe et al. 2005). Although not captured in this study, social interactions with members of the social welfare system may play a significant role in the trauma-PTSD process.

The findings relevant to personal and interpersonal agency are remarkable. They support the theoretical understanding that agency represents a process as well as an outcome (Bandura 2001), and, they highlight how clinicians may approach the treatment of PTSD in single mothering populations. CAT had a direct effect only on personal agency-specific acts that are performed intentionally to achieve a desired outcome; while PTSD symptom severity was directly affected only by interpersonal agency-purposeful communication and collaboration with people holding formal and informal power to acquire their assistance in achieving a desired outcome. Similar to Ford-Gilboe et al. (2005), the findings suggest that the oppressive nature of single mothers' traumatic experiences compromise their global beliefs about personal control by limiting the options and choices available to them. For income-assisted single mothers who do not have direct control over social conditions and institutional practices that affect their lives, the findings also highlight the need for capacity building particularly as it relates to the discontinuation of oppression interpersonal experiences and communicating/collaborating with those holding power (Smith et al. 2000). In this analysis we wanted to address the mediating effects of each variable in the trauma-PTSD process, but the demonstrated association between social strain and personal agency $(r=\bullet .47)$ and social strain and interpersonal agency $(r=\bullet .23)$ suggest that personal and interpersonal agency may mediate the relationship between social strain and the severity of mothers PTSD symptoms. Longitudinal data is needed to address the interconnection between all variables in the model.

The finding that neither frequency of visits to primary or specialized mental health service affects severity of PTSD symptoms is not all together surprising. A 3-month window may not be an adequate retrospective timeframe for assessing changes in symptom severity. There are also limitations associated with using cross sectional data to examine relationships that are dynamic in nature. While we did not address quality of care in this analysis, it would make sense that quality care in conjunction with frequent care may more effectively reduce mental health problems. These results are intriguing and justify further examinations of the relationships between quality of mental health services and PTSD in this population.

\section{Intersectional Analysis}

The sub-group analysis did not support a moderating effect of neighborhood (intersectional) status on the model. The observation that the structure of the model did not vary by neighborhood grouping, but that the strength and significance of paths varied, exemplify the difficulty in accounting for the effect of this complex construct. This analysis is a first step in accounting for the impact of intersectionality on the trauma-PTSD process; however, it may not be the best test. A longitudinal, multi-level analysis that specifically accounts for structural intersectionality (i.e. racism, social exclusion, poverty/economic disparities, resource deprivation) and political intersectionality (i.e. inequitable law, policies, directives) may be a more appropriate means of examining the ways in which intersectionality at the neighborhood level influences the trauma-PTSD process. Such an analysis would highlight oppression and/or dis/ advantage as the key factor that links the arrangement of our socially structured world to the mental health and wellbeing of people.

It must also be noted that the current observations (i.e. differences in strength and significance of paths) may be an artifact of the small sample size, particularly that of the priority group $(n=87)$. Smaller sample sizes influence both the variance in parameter estimates and the stability of fit indexes (Kline 2005). The findings may also be attributed to the fact that a larger proportion of priority neighborhood mothers immigrated to Canada. Given their history, it is possible that the trauma profile of the priority group is different from women living in non-priority neighborhoods. It is also possible that culture, ethnicity, and even the very act of immigration, influenced the meaning assigned to women's experience, their ability to resolve difficult life experiences, or access resources. Although the findings suggest the need for neighborhood-specific interventions, further validation using a larger sample and a longitudinal design is required.

\section{Implications for Practice}

As much as $60 \%$ of women in this sample report exposure to one or more assaultive or psychological trauma. We suggest that programs directed at promoting the mental 
health of single mothers receiving social assistance must address both the impact of prior violence and the prevention of future violence. Particularly when attempting to prevent future exposure, interventions will require the involvement of a number of health and social service agency that have the capacity to address violence against women and girls. The findings of this study highlight the need for social interventions that address gender-based traumas occurring in childhood and adulthood. Such interventions may utilize social marketing initiatives to educate the general public about rates of violence against women and girls, the prevailing social causes of such experiences, and the negative and long-term impact of such events.

Other interventions may focus on developing screening programs, encouraging the reporting of victimization, and managing the social and/or health impacts of violence. The trauma and social strain scale used in this study may assist clinicians in completing a thorough trauma history profile, assessing women's risk for re-exposure, and referring them to needed specialized services. Additionally, access to resources such as safe housing and a social network (formal or informal) that is diligent in promoting women's safety is essential to the recovery process.

Our findings revealed a strong association between past adverse and traumatic experiences and deterioration in the quality of women's relationships with members of their social network. The findings suggest that cumulative trauma directly and through it influence on social strain compromise women's capacity to engage in activities that allow them to achieve their personal and social goals. We suggest that when substantial cumulative trauma is identified, it should lead clinicians to assess the extent to which women are experiencing social strain as well as the extent to which their perceived sense of agency has been compromised. Clinicians need to build collaborative power sharing relationships to assist in capacity building among income-assisted single mothers. Such relationships will assist in supporting ways to discontinue oppressive interpersonal experiences while building positive ones. linterventions which support women in reducing negative social relations and in turn building positive ones; as well as those which promote their sense of personal control appear to be central to mental health promotion among this population. While this approach does not erase past trauma, we suggest it may be able to help women build the resources they need to begin addressing the impacts of trauma.

\section{Conclusion}

This study was completed with the goal of creating knowledge that may be used to promote the mental health of income-assisted single mothers. To this end, we examine a micro-and macro-level process though which mothers cumulated traumatic experiences directly and indirectly impact PTSD symptom levels. Our findings demonstrate that the trauma-PTSD process is a complex one that is determined by women's experiences and the availability or accessibility of personal, health, and social resources.

\section{References}

Afifi, T. O., Cox, B. J., \& Enns, M. W. (2006). Mental health profiles among married, never-married, and separated/divorced mothers in a nationally representative sample. Social Psychiatry and Psychiatric Epidemiology, 41(2), 122-129.

Allard, S. W., Rosen, D., \& Tolman, R. M. (2003). Access to mental health and substance abuse services among women receiving welfare in detroit. Urban Affairs Review, 38(6), 787.

Anderson, D. G., \& Rayens, M. K. (2004). Factors influencing homelessness in women. Public Health Nursing (Boston, Mass.), 21(1), 12-23.

Andrews, B., Brewin, C. R., \& Rose, S. (2003). Gender, social support, and PTSD in victims of violent crime. Journal of Traumatic Stress, 16(4), 421-427.

Arbuckle, J. L. (2006). Amos 7.0 User's Guide. Spring House: Amos Development Corporation.

Bandura, A. (2001). Social cognitive theory: an agentic perspective. Annual Review of Psychology, 52(1), 1-26.

Bassuk, E. L., Buckner, J. C., Perloff, J. N., \& Bassuk, S. S. (1998). Prevalence of mental health and substance use disorders among homeless and low-income housed mothers. The American Journal of Psychiatry, 155(11), 1561-1564.

Bassuk, E. L., Dawson, R., \& Huntington, N. (2006). Intimate partner violence in extremely poor women: longitudinal patterns and risk markers. Journal of Family Violence, 21, 387-399.

Benight, C. C., \& Bandura, A. (2004). Social cognitive theory of posttraumatic recovery: the role of perceived self-efficacy. Behaviour Research and Therapy, 42(10), 1129-1148.

Bentler, P. M. (1990). Comparative fit indexes in structural models. Psychological Bulletin, 107(2), 238-246.

Bradley, R., Schwartz, A. C., \& Kaslow, N. J. (2005). Posttraumatic stress disorder symptoms among low-income, African American women with a history of intimate partner violence and suicidal behaviors: self-esteem, social support, and religious coping. Journal of Traumatic Stress, 18(6), 685-696.

Breslau, N., Chilcoat, H. D., Kessler, R. C., Peterson, E. L., \& Lucia, V. C. (1999). Vulnerability to assaultive violence: further specification of the sex difference in post-traumatic stress disorder. Psychological Medicine, 29(4), 813-821.

Browne, M. W., \& Cudeck, R. (1993). Alternative ways of assessing model fit. In K. A. Bollen \& J. S. Long (Eds.), Testing structural equation model (3rd ed., pp. 136-162). Newbury Park: Sage.

Byrne, B. M. (2001). Structural equation modeling with AMOS. Mahwah: Lawrence Erlbaum Associates.

City of Toronto (2006). City of Toronto Priority Neighbourhoods: Overview of demographics and community services. Unpublished manuscript.

Cohen, S., Mermelstein, R., Kamarck, T., \& Hobfoll, S. E. (1985). Measuring the functional components of social support. In I. G. Sarason \& B. R. Sarason (Eds.), Social support: Theory, research, and applications (pp. 73-94). Nijhoff: Springer.

Coker, A. L., Smith, P. H., Bethea, L., King, M. R., \& McKeown, R. E. (2000). Physical health consequences of physical and 
psychological intimate partner violence. Archives of Family Medicine, 9(5), 451-457.

Coker, A. L., Smith, P. H., Thompson, M. P., McKeown, R. E., Bethea, L., \& Davis, K. E. (2002). Social support protects against the negative effects of partner violence on mental health. Journal of Women's Health \& Gender-Based Medicine, 11(5), 465-476.

Coker, A. L., Watkins, K. W., Smith, P. H., \& Brandt, H. M. (2003). Social support reduces the impact of partner violence on health: application of structural equation models. Preventive Medicine, $37(3), 259-267$.

Crenshaw, K. (1994). Mapping the margins: Intersectionality, identity politics, and violence against women of color. In $M$. A. Finemann \& R. Mykitiuk (Eds.). The public nature of private violence: The discovery of domestic abuse (pp. 93-120). New York: Routledge.

Cunradi, C. B., Caetano, R., Clark, C., \& Schafer, J. (2000). Neighborhood poverty as a predictor of intimate partner violence among White, Black, and Hispanic couples in the United States: a multilevel analysis. Annals of Epidemiology, 10(5), 297-308.

Davidson, J. R. T. (1996). Davidson Trauma Scale (DTS). North Tonawanda: Multi-Health Systems Inc.

Elhai, J. D., \& Simons, J. S. (2007). Trauma exposure and posttraumatic stress disorder predictors of mental health treatment use in college students. Psychological Services, 4(1), 3845.

Ford-Gilboe, M., Wuest, J., \& Merritt-Gray, M. (2005). Strengthening capacity to limit intrusion: theorizing family health promotion in the aftermath of woman abuse. Qualitative Health Research, 15 (4), 477-501.

Ford-Gilboe, M., Wuest, J., Merrit-Gray, M., Campbell, J. C., \& Wilk, P. (2009). Modeling the effects of intimate partner Violence and access to resources on women's health in the early years after leaving an abusive partner. Social Science Medicine, 28.

Galea, S., Ahern, J., Nandi, A., Tracy, M., Beard, J., \& Vlahov, D. (2007). Urban neighborhood poverty and the incidence of depression in a population-based cohort study. Annals of Epidemiology, 17(3), 171-179.

Ginzburg, K., Solomon, Z., Dekel, R., \& Neria, Y. (2003). Battlefield funclioning and chronic PTSD: associations with perceived self efficacy and causal attribution. Personality and Individual Differences, 34(3), 463-476.

Goldberg, L. R., \& Freyd, J. J. (2006). Self-reports of potentially traumatic experiences in an adult community sample: gender differences and test-retest stabilities of the items in a brief betrayaltrauma survey. Journal of Trauma \& Dissociation, 7(3), 39-63.

Goodwin, S. S. (1997). The marital relationship and health in women with chronic fatigue and immune dysfunction syndrome: views of wives and husbands. Nursing Research, 46(3), 138-146.

Hyman, S. M., Gold, S. N., \& Cott, M. A. (2003). Forms of social support that moderate PTSD in Childhood Sexual Abuse Survivors. Journal of Family Violence, 18(5), 295-300.

James, L. R., Mulaik, S. A., \& Brett, J. M. (1982). Causal analysis: Assumptions, models, and data. Beverley Hills: Sage.

Johansen, V. A., Wahl, A. K., Eilertsen, D. E., \& Weisaeth, L. (2007). Prevalence and predictors of post-traumatic stress disorder (PTSD) in physically injured victims of non-domestic violence. Social Psychiatry and Psychiatric Epidemiology, 42(7), 583-593.

Jöreskog, K. G., \& Sörbom, D. (1995). PRELIS: A program for multivariate data screening and data summarization. Chicago: Scientific Software International.

Kaysen, D., Resick, P. A., \& Wise, D. (2003). Living in danger: the impact of chronic traumatization and the traumatic context on posttraumatic stress disorder. Trauma, Violence \& Abuse, 4(3), 247-264.

Kline, R. B. (2005). Principles and practice of structural equation modeling. New York: The Guilford Press.
Lewis, S. F., Resnick, H. S., Ruggiero, K. J., Smith, D. W., Kilpatrick D. G., Best, C. L., et al. (2005). Assault, psychiatric diagnoses, and sociodemographic variables in relation to help-seeking behavior in a national sample of women. Journal of Traumatic Stress, 18(2), 97-105.

Lloyd, D. A., \& Turner, R. J. (2003). Cumulative adversity and posttraumatic stress disorder: evidence from a diverse community sample of young adults. The American Journal of Orthopsychiatry, 73(4), 381-391.

Pearlin, L. (1999). The stress process revisited: Reflections on conceptions and their interrelationships. In C. Aneshensel \& J. Phelan (Eds.), Handbook of the sociology of mental health (pp. 395-416). New York: Kluwer Academic/Plenum Publishers.

Pico-Alfonso, M. A. (2005). Psychological intimate partner violence: the major predictor of posttraumatic stress disorder in abused women. Neuroscience and Biobehavioral Reviews, 29(1), 181193.

Rivara, F. P., Anderson, M. L., Fishman, P., Bonomi, A. E., Reid, R. J., Carrell, D., et al. (2007). Healthcare utilization and cost for women with a history of intimate partner violence. American Journal of Preventive Medicine, 32(2), 89-96.

Samuels-Dennis, J., Ford-Gilboe, M., Avison, W. R., Wilk, P., \& Raye, S. (2009a). PTSD Symptoms Severity and Low-income Single Mother's Traumatic Experiences. Journal of Family Violence, (Under Review).

Samuels-Dennis, J., Ford-Gilboe, M., \& Bailey, A. (2009b). The Intersectionality Model of Trauma and Post-Traumatic Stress Disorder (IMT-PTSD). In O. Havinsky (Ed.), Intersectionality and health research in Canada (p. Under Review). British Columbia: University of British Columbia Press.

Schumm, J. A., Briggs-Phillips, M., \& Hobfoll, S. E. (2006). Cumulative interpersonal traumas and social support as risk and resiliency factors in predicting PTSD and depression among inner-city women. Journal of Traumatic Stress, 19(6), 825-836.

Smith, G. C., Kohn, S. J., Savage-Stevens, S. E., Finch, J. J., Ingate, R., \& Lim, Y. O. (2000). The effects of interpersonal and personal agency on perceived control and psychological wellbeing in adulthood. The Gerontologist, 40(4), 458-468.

Stein, D. J. (2004). Algorithms for primary care: an evidence-based approach to the pharmacotherapy of depression and anxiety disorders. Primary Psychiatry, 11(6), 55-78.

Stein, M. B., Walker, J. R., Hazen, A. L., \& Forde, D. R. (1997). Full and partial posttraumatic stress disorder: findings from a community survey. The American Journal of Psychiatry, 154 (8), 1114-1119.

Tilden, V. P., Nelson, C. A., \& May, B. A. (1990). The IPR inventory: development and psychometric characteristics. Nursing Research, 39(6), 337-343.

Tolman, R. M., \& Rosen, D. (2001). Domestic violence in the lives of women receiving welfare: mental health, substance dependence, and economic well-being. Violence Against Women, 7(2), 141158.

Ullman, S. E., Townsend, S. M., Filipas, H. H., \& Starzynski, L. L. (2007). Sructural models of the relationship of assault severity, social supprt, avoidance coping, self-blame, and PTSD among sexual assault survivors. Psychology of Women Quarterly, 31(1), 23.

Vranceanu, A. M., Hobfoll, S. E., \& Johnson, R. J. (2007). Child multi-type maltreatment and associated depression and PTSD symptoms: the role of social support and stress. Child Abuse \& Neglect, 31(1), 71-84.

World Health Organization (2004). The World Health Organization (WHO) World Mental Health (WMH) Survey Initiative version of the Composite International Diagnostic Interview (CIDI). Retrieved August/20, 2007, from http://www.hcp.med.harvard.edu/ wmhcidi/ftpdir_public/capi_instrument/capi_v20_pdf/CAPI\% 20V20\%20-\%20Post\%20Traumatic\%20Stress\%20Disorder.pdf. 